\title{
Magnon-polaron transport in magnetic insulators
}

\author{
Benedetta Flebus, ${ }_{1}^{1}$ Ka Shen, ${ }^{2}$ Takashi Kikkawa, ${ }^{3,4}$ Ken-ichi Uchida, ${ }^{3,5,6,7}$ Zhiyong Qiu, ${ }^{4}$ Eiji Saitoh, ${ }^{3,4,7,8}$ \\ Rembert A. Duine, ${ }^{1,9}$ and Gerrit E. W. Bauer ${ }^{2,3,4,7}$ \\ ${ }^{1}$ Institute for Theoretical Physics and Center for Extreme Matter and Emergent Phenomena, Utrecht University, Leuvenlaan 4, \\ 3584 CE Utrecht, The Netherlands \\ ${ }^{2}$ Kavli Institute of NanoScience, Delft University of Technology, Lorentzweg 1, 2628 CJ Delft, The Netherlands \\ ${ }^{3}$ Institute for Materials Research, Tohoku University, Sendai 980-8577, Japan \\ ${ }^{4}$ WPI Advanced Institute for Materials Research, Tohoku University, Sendai 980-8577, Japan \\ ${ }^{5}$ National Institute for Materials Science, Tsukuba 305-0047, Japan \\ ${ }^{6}$ PRESTO, Japan Science and Technology Agency, Saitama 332-0012, Japan \\ ${ }^{7}$ Center for Spintronics Research Network, Tohoku University, Sendai 980-8577, Japan \\ ${ }^{8}$ Advanced Science Research Center, Japan Atomic Energy Agency, Tokai 319-1195, Japan \\ ${ }^{9}$ Department of Applied Physics, Eindhoven University of Technology, P.O. Box 513, 5600 MB Eindhoven, The Netherlands
}

(Received 7 February 2017; published 14 April 2017)

\begin{abstract}
We theoretically study the effects of strong magnetoelastic coupling on the transport properties of magnetic insulators. We develop a Boltzmann transport theory for the mixed magnon-phonon modes ("magnon polarons") and determine transport coefficients and the spin diffusion length. Magnon-polaron formation causes anomalous features in the magnetic field and temperature dependence of the spin Seebeck effect when the disorder scattering in the magnetic and elastic subsystems is sufficiently different. Experimental data by Kikkawa et al. [Phys. Rev. Lett. 117, 207203 (2016)] on yttrium iron garnet films can be explained by an acoustic quality that is much better than the magnetic quality of the material. We predict similar anomalous features in the spin and heat conductivity and nonlocal spin transport experiments.
\end{abstract}

DOI: 10.1103/PhysRevB.95.144420

\section{INTRODUCTION}

The magnetoelastic coupling (MEC) between magnetic moments and lattice vibrations in ferromagnets stems from spin-orbit, dipole-dipole, and exchange interactions. This coupling gives rise to magnon-polarons, i.e., hybridized magnon and phonon modes in proximity of the intersection of the uncoupled elastic and magnetic dispersions [1-4]. Interest in the coupling of magnetic and elastic excitations emerged recently in the field of spin caloritronics [5], since it affects thermal and spin transport properties of magnetic insulators such as yttrium iron garnet (YIG) [6-11].

In this work, we address the spin Seebeck effect (SSE) at low temperatures-which provides an especially striking evidence for magnon-polarons in the form of asymmetric spikes in the magnetic field dependence [12]. The enhancement emerges at the magnetic fields corresponding to the tangential intersection of the magnonic dispersion with the acoustic longitudinal and transverse phonon branches that we explain by phase-space arguments and an unexpected high acoustic quality of YIG.

Here we present a Boltzmann transport theory for coupled magnon and phonon transport in bulk magnetic insulators and elucidate the anomalous field and temperature dependencies of the SSE in terms of the composite nature of the magnon-polarons. The good agreement between theory and the experiments generates confidence that the SSE can be used as an instrument to characterize magnons versus phonon scattering in a given material. We derive the full Onsager matrix, including spin and heat conductivity as well as the spin diffusion length. We predict magnon-polaron signatures in all transport coefficients that await experimental exposure.

This work is organized as follows. In Sec. II, we start by introducing the standard model for spin wave and phonon band dispersions of a magnetic insulator and the magnetoelastic coupling. In Sec. III, we describe the magnon-polaron modes and their field-dependent behavior in reciprocal space. The linearized Boltzmann equation is shown to lead to expressions for the magnon-polaron transport coefficients. In Sec. IV, we present numerical results for the spin Seebeck coefficient, spin and heat conductivity, and spin diffusion length for YIG. We also derive approximate analytical expressions for the field and temperature dependence of the anomalies emerging in the transport coefficients and compare our results with the experiments. In Sec. V, we present our conclusions and an outlook.

\section{MODEL}

In this section, we introduce the Hamiltonian describing the coupling between magnons and phonons in magnetic insulators. The experimentally relevant geometry is schematically depicted in Fig. 1.

\section{A. Magnetic Hamiltonian}

We consider a magnetic insulator with spins $\mathbf{S}_{p}=\mathbf{S}\left(\mathbf{r}_{p}\right)$ localized on lattice sites $\mathbf{r}_{p}$. The magnetic Hamiltonian consists of dipolar and (Heisenberg) exchange interactions between spins and of the Zeeman interaction due to an external magnetic field $\mathbf{B}=\mu_{0} H \hat{\mathbf{z}}$ [13-15]. It reads as

$$
\begin{aligned}
\mathcal{H}_{\text {mag }}= & \frac{\mu_{0}\left(g \mu_{B}\right)^{2}}{2} \sum_{p \neq q} \frac{\left|\mathbf{r}_{p q}\right|^{2} \mathbf{S}_{p} \cdot \mathbf{S}_{q}-3\left(\mathbf{r}_{p q} \cdot \mathbf{S}_{p}\right)\left(\mathbf{r}_{p q} \cdot \mathbf{S}_{q}\right)}{\left|\mathbf{r}_{p q}\right|^{5}} \\
& -J \sum_{p \neq q} \mathbf{S}_{p} \cdot \mathbf{S}_{q}-g \mu_{B} B \sum_{p} S_{p}^{z} .
\end{aligned}
$$

Here, $g$ is the $g$ factor, $\mu_{0}$ is the vacuum permeability, $\mu_{B}$ is the Bohr magneton, $J$ is the exchange interaction strength, and 
$\mathbf{r}_{p q}=\mathbf{r}_{p}-\mathbf{r}_{q}$. By averaging over the complex unit cell of a material such as YIG, we define a coarse-grained, classical spin $S=\left|\mathbf{S}_{p}\right|=a_{0}^{3} M_{s} /\left(g \mu_{B}\right)$ on a cubic lattice with unit cell lattice constant $a_{0}$, with $M_{S}$ being the zero-temperature saturation magnetization density. The crystal anisotropy is disregarded, while the dipolar interaction is evaluated for a magnetic film in the $y z$ plane, see Fig. 1. We employ the Holstein-Primakoff transformation and expand the spin operators as [16]

$$
\begin{aligned}
& S_{p}^{-}=\sqrt{2 S} a_{p}^{\dagger} \sqrt{1-\frac{a_{p}^{\dagger} a_{p}}{2 S}} \approx \sqrt{2 S}\left[a_{p}^{\dagger}-\frac{a_{p}^{\dagger} a_{p}^{\dagger} a_{p}}{4 S}\right], \\
& S_{p}^{z}=S-a_{p}^{\dagger} a_{p},
\end{aligned}
$$

where $S_{p}^{-}=S_{p}^{x}-i S_{p}^{y}$, and $a_{p} / a_{p}^{\dagger}$ annihilate/create a magnon at the lattice site $\mathbf{r}_{p}$ and obey Boson commutation rules $\left[a_{p}, a_{q}^{\dagger}\right]=\delta_{p q}$. Substituting the Fourier representation

$$
a_{p}=\frac{1}{\sqrt{N}} \sum_{\mathbf{k}} e^{i \mathbf{k} \cdot \mathbf{r}_{p}} a_{\mathbf{k}}, \quad a_{p}^{\dagger}=\frac{1}{\sqrt{N}} \sum_{\mathbf{k}} e^{-i \mathbf{k} \cdot \mathbf{r}_{p}} a_{\mathbf{k}}^{\dagger},
$$

where $N$ is the number of lattice sites, and retaining only quadratic terms in the bosonic operators and disregarding a constant, the Hamiltonian (1) becomes

$$
\mathcal{H}_{\mathrm{mag}}=\sum_{\mathbf{k}} A_{\mathbf{k}} a_{\mathbf{k}}^{\dagger} a_{\mathbf{k}}+\frac{1}{2}\left(B_{\mathbf{k}} a_{-\mathbf{k}} a_{\mathbf{k}}+B_{\mathbf{k}}^{*} a_{\mathbf{k}}^{\dagger} a_{-\mathbf{k}}^{\dagger}\right),
$$

with

$$
\begin{aligned}
& \frac{A_{\mathbf{k}}}{\hbar}=D_{\mathrm{ex}} \mathcal{F}_{\mathbf{k}}+\gamma \mu_{0} H+\frac{\gamma \mu_{0} M_{s} \sin ^{2} \theta_{\mathbf{k}}}{2}, \\
& \frac{B_{\mathbf{k}}}{\hbar}=\frac{\gamma \mu_{0} M_{s} \sin ^{2} \theta_{\mathbf{k}}}{2} e^{-2 i \phi_{\mathbf{k}}} .
\end{aligned}
$$

Here, $D_{\mathrm{ex}}=2 S J a_{0}^{2}$ is the exchange stiffness, $\gamma=g \mu_{B} / \hbar$ the gyromagnetic ratio, $\theta_{\mathbf{k}}=\arccos \left(k_{z} / k\right)$ the polar angle between wave vector $\mathbf{k}$ with $k=|\mathbf{k}|$ and the magnetic field along $\hat{\mathbf{z}}$ and $\phi_{\mathbf{k}}$ the azimuthal angle of $\mathbf{k}$ in the $x y$ plane. The form factor $\mathcal{F}(\mathbf{k})=2\left(3-\cos k_{x} a_{0}-\cos k_{y} a_{0}-\cos k_{z} a_{0}\right) / a_{0}^{2}$ can be approximated as $\mathcal{F}(\mathbf{k}) \approx k^{2}$ in the long-wavelength limit $\left(k a_{0} \ll 1\right)$. Equation (4) is diagonalized by the Bogoliubov transformation [17]

$$
\left[\begin{array}{c}
a_{\mathbf{k}} \\
a_{-\mathbf{k}}^{\dagger}
\end{array}\right]=\left[\begin{array}{cc}
u_{\mathbf{k}} & -v_{\mathbf{k}} \\
-v_{\mathbf{k}}^{*} & u_{\mathbf{k}}
\end{array}\right]\left[\begin{array}{c}
\alpha_{\mathbf{k}} \\
\alpha_{-\mathbf{k}}^{\dagger}
\end{array}\right],
$$

with parameters

$$
u_{\mathbf{k}}=\sqrt{\frac{A_{\mathbf{k}}+\hbar \omega_{\mathbf{k}}}{2 \hbar \omega_{\mathbf{k}}}}, \quad v_{\mathbf{k}}=\sqrt{\frac{A_{\mathbf{k}}-\hbar \omega_{\mathbf{k}}}{2 \hbar \omega_{\mathbf{k}}}} e^{2 i \phi_{\mathbf{k}}} .
$$

The Hamiltonian (4) is then simplified to

$$
\mathcal{H}_{\text {mag }}=\sum_{\mathbf{k}} \hbar \omega_{\mathbf{k}} \alpha_{\mathbf{k}}^{\dagger} \alpha_{\mathbf{k}},
$$

where $\hbar \omega_{\mathbf{k}}=\sqrt{A_{\mathbf{k}}^{2}-\left|B_{\mathbf{k}}\right|^{2}}$ is the magnon dispersion. For bulk magnons in the long-wavelength limit $[18,19]$,

$$
\omega_{\mathbf{k}}=\sqrt{D_{\mathrm{ex}} k^{2}+\gamma \mu_{0} H} \sqrt{D_{\mathrm{ex}} k^{2}+\gamma \mu_{0}\left(H+M_{s} \sin ^{2} \theta_{\mathbf{k}}\right)} .
$$

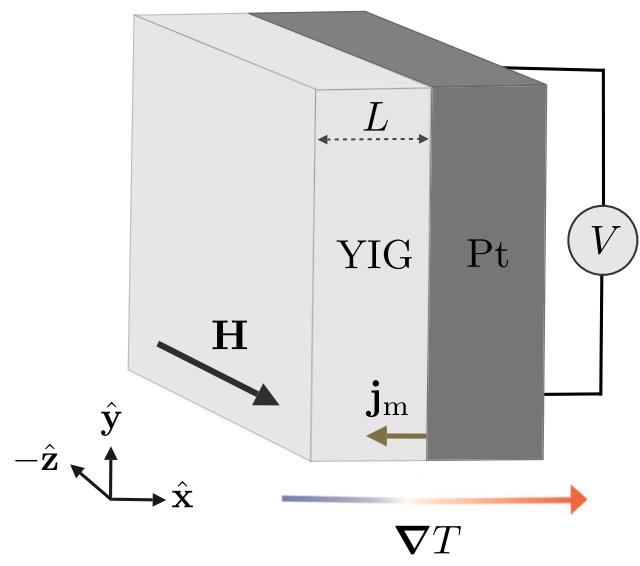

FIG. 1. Pt|YIG bilayer subject to a thermal gradient $\nabla T \| \hat{\mathbf{x}}$ and a magnetic field $\mathbf{H} \| \hat{\mathbf{z}}$. The thermal bias gives rise to a flow of magnons, i.e., a magnonic spin current $\mathbf{j}_{\mathrm{m}}$, in the YIG film of thickness $L$. In the Pt lead, the spin current is then converted into a measurable voltage $V$ via the inverse spin Hall effect.

We disregard Damon-Eshbach modes [20] localized at the surface since, in the following, we focus on transport in thick films normal to the plane, i.e., in the $x$ direction in Fig. 1. For thick films, the backward moving volume modes are relevant only for wave numbers $k$ very close to the origin and are disregarded as well. Higher-order terms in the magnon operators that encode magnon-magnon scattering processes have been disregarded as well in Eq. (4), which is allowed for sufficiently low magnon-densities or temperatures (for YIG $\lesssim 100 \mathrm{~K}$ [21]). In this regime, the main relaxation mechanism is magnon scattering by static disorder [6] with Hamiltonian

$$
\mathcal{H}_{\text {mag-imp }}=\sum_{\mathbf{k}, \mathbf{k}^{\prime}} v_{\mathbf{k}, \mathbf{k}^{\prime}}^{\mathrm{mag}} \alpha_{\mathbf{k}}^{\dagger} \alpha_{\mathbf{k}^{\prime}}
$$

where $v_{\mathbf{k}, \mathbf{k}^{\prime}}^{\mathrm{mag}}$ is an impurity-scattering potential. In the following, we employ the isotropic, short-range scattering approximation $v_{\mathbf{k}, \mathbf{k}^{\prime}}^{\mathrm{mag}}=v^{\mathrm{mag}}$.

\section{B. Mechanical Hamiltonian}

We focus on lattice vibrations or sound waves with wavelengths much larger than the lattice constant that are well-described by continuum mechanics. The Hamiltonian of an elastically isotropic solid reads [22]

$$
\begin{aligned}
\mathcal{H}_{\mathrm{el}}= & \int d^{3} r \sum_{i, j} \frac{\Pi_{i}^{2}(\mathbf{r})}{2 \bar{\rho}} \delta_{i j}+\left(c_{\|}^{2}-c_{\perp}^{2}\right) \frac{\bar{\rho}}{2} \frac{\partial R_{i}(\mathbf{r})}{\partial x_{i}} \frac{\partial R_{j}(\mathbf{r})}{\partial x_{j}} \\
& +c_{\perp}^{2} \frac{\bar{\rho}}{2} \frac{\partial R_{i}(\mathbf{r})}{\partial x_{j}} \frac{\partial R_{i}(\mathbf{r})}{\partial x_{j}}
\end{aligned}
$$

where $\bar{\rho}$ is the average mass density, $R_{i}$ is the $i$ th component of the displacement vector $\mathbf{R}$ of a volume element at $\mathbf{r}$ with respect to its equilibrium position, $\Pi_{i}$ is the conjugate phonon momentum, and $c_{\|}$and $c_{\perp}$ are the velocities of the longitudinal acoustic (LA) and transverse acoustic (TA) lattice waves, respectively. The Hamiltonian (11) can be quantized by the 
phonon creation (annihilation) operators $c_{\lambda \mathbf{k}}^{\dagger}\left(c_{\lambda \mathbf{k}}\right)$ as

$$
\begin{aligned}
& R_{i}(\mathbf{r}, t)=\sum_{\mathbf{k}, \lambda} \epsilon_{i \lambda}(\mathbf{k})\left(\frac{\hbar}{2 \bar{\rho} V \omega_{\lambda \mathbf{k}}}\right)^{1 / 2}\left(c_{\lambda \mathbf{k}}^{\dagger}+c_{\lambda-\mathbf{k}}\right) e^{i \mathbf{k r}} \\
& \Pi_{i}(\mathbf{r}, t)=i \sum_{\mathbf{k}, \lambda} \epsilon_{i \lambda}(\mathbf{k})\left(\frac{\bar{\rho} \hbar \omega_{\lambda \mathbf{k}}}{2 V}\right)^{1 / 2}\left(c_{\lambda \mathbf{k}}^{\dagger}-c_{\lambda-\mathbf{k}}\right) e^{-i \mathbf{k r}}
\end{aligned}
$$

where $\lambda=1,2$ labels the shear waves polarized normal to the wave vector $\mathbf{k}$ (TA phonons), while $\lambda=3$ represents a pressure wave (LA phonons). Here, $\omega_{\lambda \mathbf{k}}=c_{\lambda}|\mathbf{k}|$ is the phonon dispersion and $\epsilon_{i \lambda}(\mathbf{k})=\hat{\mathbf{x}}_{i} \cdot \hat{\epsilon}(\mathbf{k}, \lambda)$ are Cartesian components $i=x, y, z$ of the unit polarization vectors

$$
\begin{aligned}
& \hat{\epsilon}(\mathbf{k}, 1)=\left(\cos \theta_{\mathbf{k}} \cos \phi_{\mathbf{k}}, \cos \theta_{\mathbf{k}} \sin \phi_{\mathbf{k}},-\sin \theta_{\mathbf{k}}\right), \\
& \hat{\epsilon}(\mathbf{k}, 2)=i\left(-\sin \phi_{\mathbf{k}}, \cos \phi_{\mathbf{k}}, 0\right), \\
& \hat{\epsilon}(\mathbf{k}, 3)=i\left(\sin \theta_{\mathbf{k}} \cos \phi_{\mathbf{k}}, \sin \theta_{\mathbf{k}} \sin \phi_{\mathbf{k}}, \cos \theta_{\mathbf{k}}\right),
\end{aligned}
$$

that satisfy $\hat{\epsilon}^{*}(\mathbf{k}, \lambda)=\hat{\epsilon}(-\mathbf{k}, \lambda)[6]$. In terms of the operators $c_{\lambda \mathbf{k}}$ and $c_{\lambda \mathbf{k}}^{\dagger}$, Eq. (11) becomes

$$
\mathcal{H}_{\mathrm{el}}=\sum_{\mathbf{k}, \lambda} \hbar \omega_{\lambda \mathbf{k}}\left(c_{\lambda \mathbf{k}}^{\dagger} c_{\lambda \mathbf{k}}+\frac{1}{2}\right)
$$

Analogous to magnons, at low temperatures, phonon relaxation is dominated by static disorder:

$$
\mathcal{H}_{\text {imp }}=\sum_{\lambda} \sum_{\mathbf{k}, \mathbf{k}^{\prime}} v_{\mathbf{k}, \mathbf{k}^{\prime}}^{\mathrm{ph}} c_{\lambda \mathbf{k}}^{\dagger} c_{\lambda \mathbf{k}^{\prime}},
$$

where $v_{\mathbf{k}, \mathbf{k}^{\prime}}^{\mathrm{ph}}$ is the phonon impurity-scattering potential, in the following assumed to be isotropic and short-range, i.e., $v_{\mathbf{k}, \mathbf{k}^{\prime}}^{\mathrm{ph}}=v^{\mathrm{ph}}$.

\section{Magnetoelastic coupling}

The magnetic excitations are coupled to the elastic displacement via magnetoelastic interactions. In the long-wavelength limit, to leading order in the magnetization $M_{i}=n g \mu_{B} S_{i}$ ( $n=1 / a_{0}^{3}$ ) and displacement field $R_{i}$, the magnetoelastic energy reads as [2,17]

$$
\begin{aligned}
\mathcal{H}_{\text {mec }}= & \frac{\hbar n}{M_{s}^{2}} \int d^{3} r \sum_{i j}\left[B_{i j} M_{i}(\mathbf{r}) M_{j}(\mathbf{r})\right. \\
& \left.+B_{i j}^{\prime} \frac{\partial \mathbf{M}(\mathbf{r})}{\partial r_{i}} \cdot \frac{\partial \mathbf{M}(\mathbf{r})}{\partial r_{j}}\right] R_{i j}(\mathbf{r}),
\end{aligned}
$$

where $B_{i j}=\delta_{i j} B_{\|}+\left(1-\delta_{i j}\right) B_{\perp} \quad$ and $\quad B_{i j}^{\prime}=\delta_{i j} B_{\|}^{\prime}+(1-$ $\left.\delta_{i j}\right) B_{\perp}^{\prime}$ are the phenomenological magnetoelastic constants and

$$
R_{i j}(\mathbf{r})=\frac{1}{2}\left[\frac{\partial R_{i}(\mathbf{r})}{\partial r_{j}}+\frac{\partial R_{j}(\mathbf{r})}{\partial r_{i}}\right],
$$

is the displacement gradient $R_{i j}$.

The exchange term $\sim B_{i j}^{\prime}$ in Eq. (17) contains magnetization gradients and predominantly affects short wavelength magnons. We disregard this term since we are interested in capturing low temperature features. Linearizing with respect to small nonequilibrium variables- $R_{i}, M_{x}, M_{y}-\mathrm{Eq}$. (17) then becomes

$$
\begin{aligned}
\mathcal{H}_{\text {mec }}= & \hbar n B_{\perp}\left(\frac{\gamma \hbar^{2}}{4 M_{s} \bar{\rho}}\right)^{1 / 2} \sum_{\mathbf{k}, \lambda} k \omega_{\mathbf{k} \lambda}^{-1 / 2} e^{-i \phi} a_{\mathbf{k}}\left(c_{\lambda-\mathbf{k}}+c_{\lambda \mathbf{k}}^{\dagger}\right) \\
& \times\left(-i \delta_{\lambda 1} \cos 2 \theta_{\mathbf{k}}+i \delta_{\lambda 2} \cos \theta_{\mathbf{k}}-\delta_{\lambda 3} \sin 2 \theta_{\mathbf{k}}\right)+\text { H.c. },
\end{aligned}
$$

where $\delta_{\lambda i}$ is the Kronecker delta.

\section{MAGNON-POLARONS}

Here we introduce magnon-polarons and formulate their semiclassical transport properties.

\section{A. Magnon-polaron modes}

We rewrite the Hamiltonian $\mathcal{H}=\mathcal{H}_{\text {mag }}+\mathcal{H}_{\mathrm{el}}+\mathcal{H}_{\text {mec }}$ as

$$
\mathcal{H}=\frac{1}{2} \sum_{\mathbf{k}}\left[\begin{array}{ll}
\boldsymbol{\beta}_{\mathbf{k}}^{\dagger} & \boldsymbol{\beta}_{-\mathbf{k}}
\end{array}\right] \cdot \boldsymbol{H}_{\mathbf{k}} \cdot\left[\begin{array}{ll}
\boldsymbol{\beta}_{\mathbf{k}} & \boldsymbol{\beta}_{-\mathbf{k}}^{\dagger}
\end{array}\right]^{T},
$$

where $\boldsymbol{\beta}_{\mathbf{k}}^{\dagger} \equiv\left(\alpha_{\mathbf{k}}^{\dagger} c_{1 \mathbf{k}}^{\dagger} c_{2 \mathbf{k}}^{\dagger} c_{3 \mathbf{k}}^{\dagger}\right)$ and the Bogoliubov-de Gennes Hamiltonian $\boldsymbol{H}_{\mathbf{k}}$ is an $8 \times 8$ Hermitian matrix. Following Ref. [23], we introduce the para-unitary matrix $\mathcal{T}_{\mathbf{k}}$ that diagonalizes $\boldsymbol{H}_{\mathbf{k}}$ as

$$
\boldsymbol{H}_{\mathrm{k}} \mathcal{T}_{\mathbf{k}}=v \mathcal{T}_{\mathbf{k}}\left[\begin{array}{cc}
E_{\mathrm{k}} & \mathbf{0} \\
\mathbf{0} & -E_{-\mathbf{k}}
\end{array}\right],
$$

where $[\boldsymbol{v}]_{j m}=\delta_{j m} v_{j}$ with $v_{j}=+1$ for $j=1, \ldots, 4$ and $v_{j}=$ -1 for $j=5, \ldots, 8$, and $\boldsymbol{E}_{\mathbf{k}}$ is a diagonal matrix, whose $i$ th element $\hbar \Omega_{i \mathbf{k}}$ represents the dispersion relation of the hybrid mode with creation operator $\Gamma_{i \mathbf{k}}^{\dagger}=\sum_{j=1}^{8}\left[\boldsymbol{\beta}_{\mathbf{k}}^{\dagger} \boldsymbol{\beta}_{-\mathbf{k}}\right]_{j}\left(\mathcal{\mathcal { T }}_{\mathbf{k}}^{-1}\right)_{i j}^{*}$ that is neither a pure phonon or magnon, but a magnon-polaron.

Let us focus our attention to waves propagating perpendicularly to the magnetic field, i.e., $\mathbf{k}=k \hat{\mathbf{x}}$ (see Fig. 1). It follows from Eq. (19) that magnon-polarons involve only TA phonons. Disregarding the dipolar interactions, the TA phonon branch is tangent to the magnon dispersion for $\mu_{0} H_{\perp}=c_{\perp}^{2} / 4 D_{e x} \gamma$ at $k_{\perp}=c_{\perp} / 2 D_{e x}$. This estimate holds for $M_{s} \ll H_{\perp}$; otherwise the dipolar interaction shifts the magnon dispersion to higher values, leading to a smaller critical field $H_{\perp}$. For $H<H_{\perp}$, the TA phonon dispersion intersects the spin wave spectrum at two crossing points, $k_{1}$ and $k_{2}$,

$$
k_{1,2}=k_{\perp} \mp \sqrt{k_{\perp}^{2}-\frac{\gamma \mu_{0} H}{D_{e x}}},
$$

where the minus (plus) corresponds to the label 1 (2). In the vicinity of $k_{1,2}$, the modes corresponding to the dispersions $\Omega_{1,2 k}$ are strongly coupled, as shown in the inset of Fig. 2(a). The magnetoelastic coupling changes the crossing at $k_{1,2}$ into an anticrossing with energy splitting $\Delta \Omega_{k_{1,2}}=\Omega_{2 k_{1,2}}-\Omega_{1 k_{1,2}}$. For $k \ll k_{1}$, the $\Gamma_{1 k}^{\dagger}\left(\Gamma_{2 k}^{\dagger}\right)$ mode resembles closely a pure spin wave (lattice vibration) whilst for $k_{1} \ll k \ll k_{2}$ these roles are reversed, returning to their original character for $k \gg k_{2}$. At the critical magnetic field $H_{\perp}$, the magnon dispersion shifts upwards such that the TA phonon branch becomes tangential. Figure 2(b) shows that this "touching" condition generates the strongest effects of the MEC, since the magnon and phonon modes are strongly coupled over a relatively large volume in 

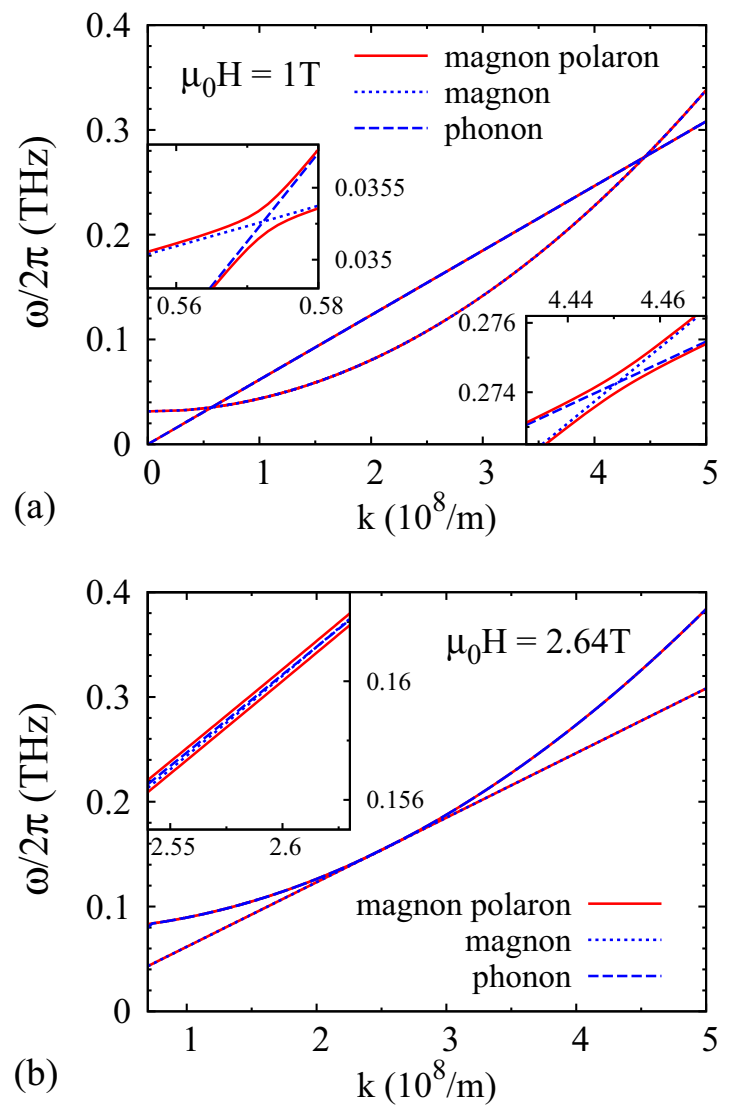

FIG. 2. Magnon, TA phonon $(\lambda=1)$, and magnon-polaron mode dispersions for YIG (see Table I for parameters) with $\mathbf{H} \| \hat{\mathbf{z}}$ and $\mathbf{k} \| \hat{\mathbf{x}}$ $(\theta=\pi / 2$ and $\phi=0)$. (a) For $\mu_{0} H=1 \mathrm{~T}$, the magnon and transverse phonon dispersions intersect at two crossing points $\mathrm{k}_{1,2}$. The mixing between magnons and phonons (see insets) is maximized at these crossings. (b) For $\mu_{0} H_{\perp}=2.64 \mathrm{~T}$, the phonon dispersion becomes a tangent to the magnon dispersion which maximizes the phase space of magnon-polaron formation (see inset).

momentum space. At higher magnetic fields, the uncoupled magnonic and TA phononic curves no longer cross, hence the MEC does not play a significant role, and $\mathcal{T}_{k}$ reduces to the identity matrix. An analogous physical picture holds when considering the magnon-polaron modes arising from the coupling between magnons and LA phonons for $\sin 2 \theta_{\mathbf{k}} \neq 0$, with critical field $\mu_{0} H_{\|}=c_{\|}^{2} / 4 D_{e x} \gamma$ and touch point $k_{\|}=c_{\|} / 2 D_{e x}\left(\right.$ for $\left.M_{s} \ll H_{\|}\right)$.

\section{B. Magnon-polaron transport}

We proceed to assess the magnetoelastic coupling effects on the transport properties of a magnetic insulator in order to model the spin Seebeck effect and magnon injection by heavy metal contacts. A nonequilibrium state at the interface between the magnetic insulator and the normal metal generates a spin current that can be detected by the inverse spin Hall effect, as shown in Fig. 1. The spin current and spin-mediated heat currents are then proportional to the interface spin mixing conductance that is governed by the exchange interaction between conduction electrons in the metal and the magnetic order in the ferromagnet. In the presence of magnon-polarons, the excitations at the interface have mixed character. Since the spin-pumping and spin torque processes are mediated by the exchange interaction, only the magnetic component of the magnon-polaron in the metal interacts with the conduction electrons. We focus here on the limit in which the smaller of the magnon spin diffusion length and magnetic film thickness is sufficiently large such that the spin current is dominated by the bulk transport and the interface processes may be disregarded. We therefore calculate in the following the spin-projected angular momentum and heat currents in the bulk of the ferromagnet, assuming that the interface scattering processes and subsequent conversion into an inverse spin Hall voltage do not change the dependence of the observed signals on magnetic field, temperature gradient, material parameters, etc.

Since the phonon specific heat is an order of magnitude larger than the magnon one at low temperatures [24], we may assume that the phonon temperature and distribution is not significantly perturbed by the magnons. $T$ is the phonon temperature at equilibrium and we are interested in the response to a constant gradient $\nabla T \| \hat{\mathbf{x}}$. The spinconserving relaxation of the magnon distribution towards the phonon temperature is assumed to be so efficient that the magnon temperature is everywhere equal to the phonon temperature. Also the magnon-polaron temperature profile is then $T(x)=T+|\nabla T| x$. Assuming efficient thermalization of both magnons and phonons and weak spin-non-conserving processes as motivated by the small Gilbert damping, a nonequilibrium distribution as injected by a metallic contact can be parameterized by a single parameter, viz. the effective magnonpolaron chemical potential $\mu$ [33]. This approximation might break down at a very low temperatures, but to date there is no evidence for that.

In equilibrium, the chemical potential of magnons and phonons vanishes since their number is not conserved. The occupation of the $i$ th magnon-polaron in equilibrium is therefore given by the Planck distribution function

$$
f_{i \mathbf{k}}^{(0)}=\left(\exp \frac{\hbar \Omega_{i \mathbf{k}}}{k_{B} T}-1\right)^{-1} .
$$

Note that here we have assumed the $i$ th magnon polaron scattering rate to be sufficiently smaller than the gap between the magnon-polaron mode dispersions, i.e., $\tau_{i \mathbf{k}_{i}}^{-1} \ll \Delta \Omega_{\mathbf{k}_{i}}$ for every $\mathbf{k}_{i}$, which guarantees the $i$ th magnon-polaron to not dephase and hence its distribution function to be well-defined. We focus on films with thickness $L \gg \Lambda_{\mathrm{mag}}, \Lambda_{\mathrm{ph}, \lambda}, \ell_{\mathrm{m}}, \ell_{\mathrm{ph}, \lambda}$, where $\Lambda_{\mathrm{mag}}=\left(4 \pi \hbar D_{e x} / k_{B} T\right)^{1 / 2}$ and $\Lambda_{\mathrm{ph}, \lambda}=\hbar c_{\lambda} / k_{B} T$ are the thermal magnon and phonon (de Broglie) wavelengths, respectively, and $\ell_{\mathrm{m}}\left(\ell_{\mathrm{ph}, \lambda}\right)$ the magnon (phonon) mean free path. The bulk transport of magnon-polarons is then semiclassical and can be treated by means of Boltzmann transport theory. In the relaxation time approximation to the collision integral, the Boltzmann equation for the out-of-equilibrium distribution function $f_{i \mathbf{k}}(\mathbf{r}, t)$ reads

$$
\partial_{t} f_{i \mathbf{k}}+\partial_{\mathbf{r}} f_{i \mathbf{k}} \cdot \partial_{\mathbf{k}} \Omega_{i \mathbf{k}}=-\left(f_{i \mathbf{k}}-f_{i \mathbf{k}}^{(0)}\right) / \tau_{i \mathbf{k}},
$$

where $\tau_{i \mathbf{k}}$ is the relaxation time towards equilibrium. In the steady state, the deviation $\delta f_{i \mathbf{k}}(\mathbf{r})=f_{i \mathbf{k}}(\mathbf{r})-f_{i \mathbf{k}}^{(0)}$ encodes the 
magnonic spin, $\mathbf{j}_{\mathrm{m}}$, and heat, $\mathbf{j}_{Q, \mathrm{~m}}$, current densities

$$
\begin{gathered}
\mathbf{j}_{\mathrm{m}}=\int \frac{d^{3} \mathbf{k}}{(2 \pi)^{3}} \sum_{i} W_{i \mathbf{k}}\left(\partial_{\mathbf{k}} \Omega_{i \mathbf{k}}\right) \delta f_{i \mathbf{k}}, \\
\mathbf{j}_{Q, \mathrm{~m}}=\int \frac{d^{3} \mathbf{k}}{(2 \pi)^{3}} \sum_{i} W_{i \mathbf{k}}\left(\partial_{\mathbf{k}} \Omega_{i \mathbf{k}}\right)\left(\hbar \Omega_{i \mathbf{k}}\right) \delta f_{i \mathbf{k}} .
\end{gathered}
$$

Here, $W_{i \mathbf{k}}=\left|\left(\boldsymbol{U}_{\mathbf{k}}\right)_{i 1}\right|^{2}+\left|\left(\boldsymbol{U}_{\mathbf{k}}\right)_{i 5}\right|^{2}$ is the magnetic amplitude of the $i$ th quasiparticle branch with $\boldsymbol{U}_{\mathbf{k}}=\mathcal{T}_{\mathbf{k}}^{-1}$. For small temperature gradients, Eqs. (25) and (26) can be linearized

$$
\begin{gathered}
\mathbf{j}_{\mathrm{m}} \simeq-\boldsymbol{\sigma} \cdot \nabla \mu-\zeta \cdot \nabla T, \\
\mathbf{j}_{Q, \mathrm{~m}} \simeq-\boldsymbol{\rho}^{(\mathrm{m})} \cdot \nabla \mu-\boldsymbol{\kappa}^{(\mathrm{m})} \cdot \nabla T,
\end{gathered}
$$

where the tensors $\sigma, \kappa^{(\mathrm{m})}, \zeta$, and $\rho^{(\mathrm{m})}(=T \zeta$ by the OnsagerKelvin relation) are, respectively, the spin and (magnetic) heat conductivities, and the spin Seebeck and Peltier coefficients. In the absence of magnetoelastic coupling, Eqs. (27) and (28) reduce to the spin and heat currents of magnon diffusion theory [33].

The total heat current $\mathbf{j}_{Q}$ carried by both magnon and phonon systems does not invoke the spin projection $W_{i \mathbf{k}}$, i.e.,

$$
\begin{aligned}
\mathbf{j}_{Q} & =\int \frac{d^{3} \mathbf{k}}{(2 \pi)^{3}} \sum_{i}\left(\partial_{\mathbf{k}} \Omega_{i \mathbf{k}}\right)\left(\hbar \Omega_{i \mathbf{k}}\right) \delta f_{i \mathbf{k}}, \\
& \simeq-\boldsymbol{\kappa} \cdot \nabla T,
\end{aligned}
$$

where $\boldsymbol{\kappa}$ is the total heat conductivity.

In terms of the general transport coefficients

$$
\begin{aligned}
L_{\alpha \gamma}^{m n}= & \beta \int \frac{d^{3} \mathbf{k}}{(2 \pi)^{3}} \sum_{i}\left(W_{i \mathbf{k}}\right)^{m} \tau_{i \mathbf{k}}\left(\partial_{k_{\alpha}} \Omega_{i \mathbf{k}}\right)\left(\partial_{k_{\gamma}} \Omega_{i \mathbf{k}}\right) \\
& \times \frac{e^{\beta \hbar \Omega_{i \mathbf{k}}}}{\left(e^{\beta \hbar \Omega_{i \mathbf{k}}}-1\right)^{2}}\left(\hbar \Omega_{i \mathbf{k}}\right)^{n},
\end{aligned}
$$

(with $\beta=1 / k_{B} T$ ), we identify $\sigma_{\alpha \gamma}=L_{\alpha \gamma}^{10}, \zeta_{\alpha \gamma}=L_{\alpha \gamma}^{11} / T$, $\kappa_{\alpha \gamma}^{(\mathrm{m})}=L_{\alpha \gamma}^{12} / T$ and $\kappa_{\alpha \gamma}=L_{\alpha \gamma}^{02} / T$.

At low temperatures, the excitations relax dominantly by elastic magnon- and phonon-disorder scattering as modelled here by Eqs. (10) and (16), respectively. The Fermi golden rule scattering rate $\tau_{i \mathbf{k}}^{-1}$ of the $i$ th magnon-polaron reads

$$
\begin{aligned}
\tau_{i \mathbf{k}}^{-1}= & \frac{2 \pi}{\hbar} \sum_{l=1}^{4} \sum_{j \mathbf{k}^{\prime}}\left[\left(\boldsymbol{U}_{\mathbf{k}^{\prime}}\right)_{j l}^{*}\left(\boldsymbol{U}_{\mathbf{k}}\right)_{i l}\right. \\
& \left.+\left(\boldsymbol{U}_{\mathbf{k}^{\prime}}\right)_{j l+4}^{*}\left(\boldsymbol{U}_{\mathbf{k}}\right)_{i l+4}\right]^{2}\left|v_{l}\right|^{2} \delta\left(\hbar \Omega_{i \mathbf{k}}-\hbar \Omega_{j \mathbf{k}^{\prime}}\right),
\end{aligned}
$$

where $v_{1}=v^{\text {mag }}$ and $v_{2,3,4}=v^{\mathrm{ph}}$, while the purely magnonic and phononic scattering rates are given by

$$
\tau_{\mathbf{k}, \text { mag }}^{-1}=\frac{L^{3}\left|v^{\text {mag }}\right|^{2}}{2 \pi \hbar^{2} D_{e x}} k, \quad \tau_{\mathbf{k}, \mathrm{ph}_{\lambda}}^{-1}=\frac{L^{3}\left|v^{\mathrm{ph}}\right|^{2}}{\pi \hbar^{2} c_{\lambda}} k^{2} .
$$

\section{RESULTS}

In this section, we discuss our numerical results for the transport coefficients, in particular, the emergence of field and temperature dependent anomalies, and we compare the
TABLE I. Selected YIG parameters [25-32].

\begin{tabular}{lccc}
\hline \hline & Symbol & Value & Unit \\
\hline Macrospin & $\mathrm{S}$ & 20 & - \\
$g$ factor & $\mathrm{g}$ & 2 & - \\
Lattice constant & $a_{0}$ & 12.376 & $\AA$ \\
Gyromagnetic ratio & $\gamma$ & $2 \pi \times 28$ & $\mathrm{GHz} \mathrm{T}^{-1}$ \\
Saturation magnetization & $\mu_{0} M_{s}$ & 0.2439 & $\mathrm{~T}$ \\
Exchange stiffness & $D_{e x}$ & $7.7 \times 10^{-6}$ & $\mathrm{~m}^{2} \mathrm{~s}^{-1}$ \\
LA-phonon sound velocity & $c_{\|}$ & $7.2 \times 10^{3}$ & $\mathrm{~m} \mathrm{~s}^{-1}$ \\
TA-phonon sound velocity & $c_{\perp}$ & $3.9 \times 10^{3}$ & $\mathrm{~m} \mathrm{~s}^{-1}$ \\
Magnetoelastic coupling & $B_{\perp}$ & $2 \pi \times 1988$ & $\mathrm{GHz}^{-3}$ \\
Average mass density & $\bar{\rho}$ & $5.17 \times 10^{3}$ & $\mathrm{Kg} \mathrm{m}^{-3}$ \\
Gilbert damping & $\alpha$ & $10^{-4}$ & - \\
\hline \hline
\end{tabular}

thermally induced spin current with measured spin Seebeck voltages [12].

\section{A. Spin and heat transport}

We consider a sufficiently thick ( $>1 \mu \mathrm{m})$ YIG film subject to a temperature gradient $\nabla T \| \hat{\boldsymbol{x}}$ and magnetic field $\mathbf{H} \| \hat{\boldsymbol{z}}$, as illustrated in Fig. 1. The parameters we employ are summarized in Table I. A scattering potential $\left|v^{\mathrm{mag}}\right|^{2}=10^{-5} \mathrm{~s}^{-2}$ (with $v^{\mathrm{mag}}$ in units of $\hbar$ ) reproduces the observed low-temperature magnon mean free path [24]. We treat the ratio between magnetic and nonmagnetic impurity-scattering potentials, $\eta=$ $\left|v^{\mathrm{mag}} / v^{\mathrm{ph}}\right|^{2}$, as an adjustable parameter. With the deployed scattering potentials $\tau_{\mathbf{k}_{i}}^{-1} \ll \Delta \Omega_{\mathbf{k}_{i}}$ for all magnon-polaron modes, ensuring the validity of our treatment. We compute the integrals appearing in Eq. (30) numerically on a fine grid ( $\sim 10^{6} k$ points) to guarantee accurate results.

Figure 3(a) shows the magnon-polaron scattering times and how they deviate from the purely phononic and magnonic ones close to the anticrossings. At the "touching" fields, the phase space portion over which the scattering times are modified with respect to the uncoupled situation is maximal [see Fig. 2(b)] as are the effects on spin and heat transport properties as discussed below.

In Fig. 4, we plot the (bulk) spin Seebeck coefficient $\zeta_{x x}$ as a function of magnetic field for different values of $\eta$. For $\eta=1, \zeta_{x x}$ decreases monotonously with increasing magnetic field, while for $\eta \neq 1$ two anomalies are observed at $\mu_{0} H_{\perp} \sim 2.64 \mathrm{~T}$ and $\mu_{0} H_{\|} \sim 9.3 \mathrm{~T}$. More precisely, peaks (dips) appear for $\eta=100(0.01)$ at the same magnetic fields but with amplitudes that depend on temperature.

The underlying physics can be understood in terms of the dispersion curves plotted in the inset of Fig. 5(a). The first (second) anomaly occurs when the TA (LA) phonon branch becomes a tangent of the magnon dispersion, which maximizes the integrated magnon-polaron coupling.

The group velocity of the resulting magnon-polaron does not differ substantially from the purely magnonic one, but its scattering time can be drastically modified, depending on the ratio between the magnonic and phononic scattering potentials [see Fig. 3(b)]. The spin currents can therefore be both enhanced or suppressed by the MEC. When the magnonimpurity scattering potential is larger than the phonon-impurity one, the hybridization induced by the MEC lowers the 

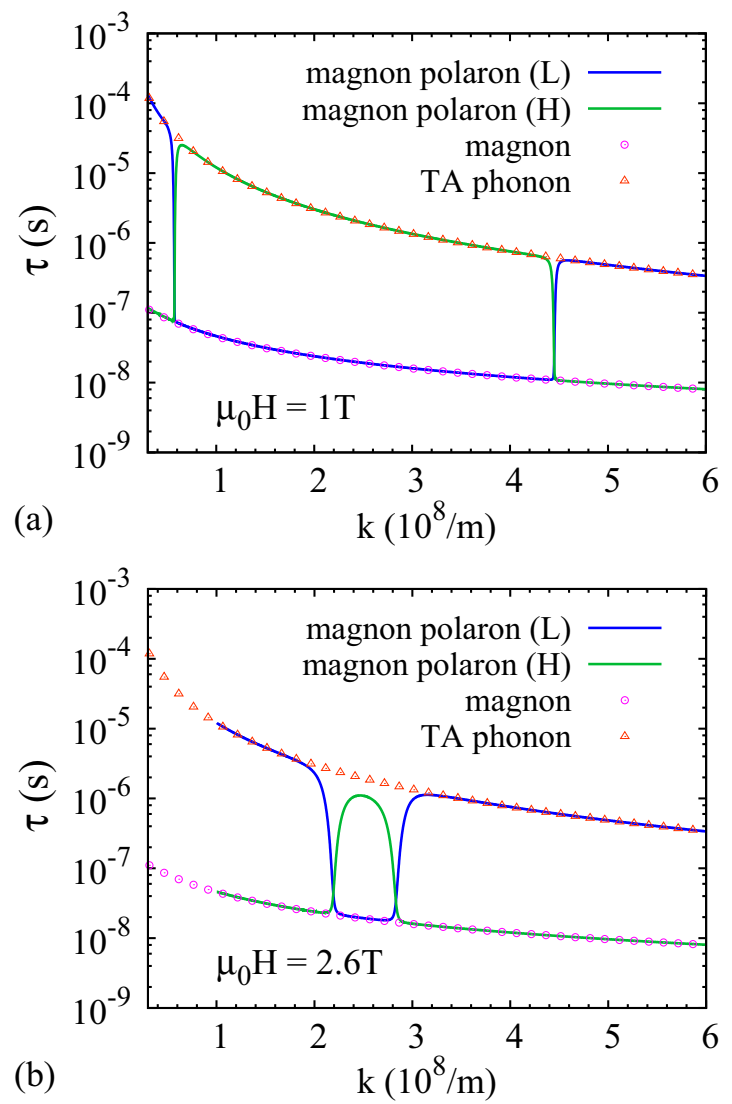

FIG. 3. (a) Scattering times of magnons, TA phonons $(\lambda=1)$, and lower (L)/upper (H) branch magnon-polarons in YIG for $\mu_{0} H=1 \mathrm{~T}$ $(\mathbf{H} \| \hat{\mathbf{z}})$ as a function of wave vector $\mathbf{k} \| \hat{\mathbf{x}}$ for $\eta=100$. (b) Same as (a) but $\mu_{0} H_{\perp}=2.64 \mathrm{~T}$.

effective potential perceived by magnons, giving rise to an enhanced scattering time and hence larger currents. This can be confirmed by comparing the blue solid $(\eta=100)$ and the black dash-dotted $\left(\eta=10^{3}\right)$ lines in Fig. 5(a), showing that the magnitude of the peaks increases with increasing $\eta$. When magnetic and nonmagnetic scattering potentials are the same, i.e., $\eta=1$, the anomalies vanish as illustrated by the dashed blue line in Fig. 5(a), and agrees with the results obtained in the absence of MEC (triangles).

The frequencies at which magnon and phonon dispersions are tangential for uncoupled transverse and longitudinal modes are $0.16 \mathrm{THz}(\hat{=} 8 \mathrm{~K}$ ) and $0.53 \mathrm{THz}(\hat{=} 26 \mathrm{~K})$. Far below these temperatures, the magnon-polaron states are not populated, which explains the disappearance of the second anomaly and the strongly reduced magnitude of the first one at $1 \mathrm{~K}$ in Fig. 4. In the opposite limit, the higher-energy anomaly becomes relatively stronger [see the solid curve at $50 \mathrm{~K}$ in Fig. 4]. The overall decay of the spin Seebeck coefficient with increasing magnetic field is explained by the freeze-out caused by the increasing magnon gap opened by the magnetic field [see the inset of Fig. 5(a)].

This strong decrease has been observed in single YIG crystals [34,35], but it is suppressed in thinner samples or even enhanced at low temperatures [12]. The effect is tentatively ascribed to the paramagnetic GGG substrate that becomes magnetically active a low temperatures [12] and is

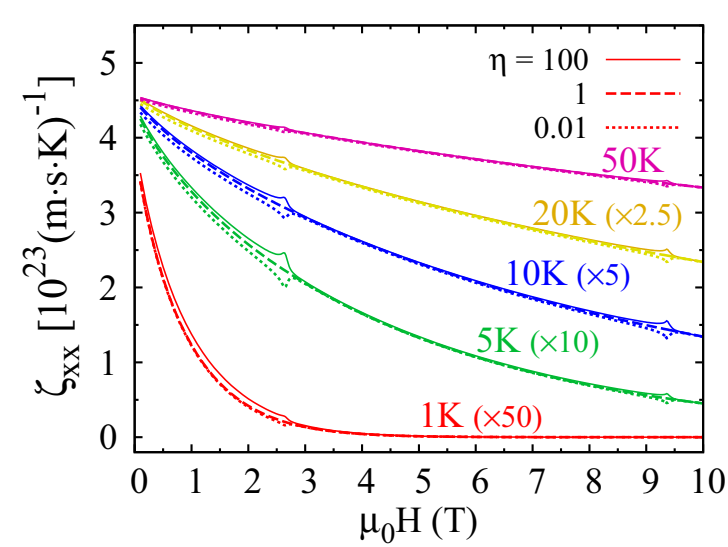

FIG. 4. Magnetic field and temperature dependence of the spin Seebeck coefficient $\zeta_{x x}$ for different values of the ratio $\eta$ between magnon and phonon impurity-scattering potentials.

beyond the scope of the present theory. We therefore subtract the pure magnonic background [triangles in Fig. 5(a)] from the magnon-polaron spin currents, which leads to the net magnon-polaron contribution shown in Fig. 5(b).

The dipolar interaction is responsible for the anisotropy in the magnon dispersion in Eq. (9), which is reflected in the magnetic field dependence of the heat and spin currents. In Fig. 5(c), we plot $\zeta_{x x}$ as function of the angle $\vartheta_{T}$ between magnetic field and transport direction for $\eta=100$ and $T=$ $10 \mathrm{~K}$. The magnon-polaron contributions for magnetization parallel and perpendicular to the transport are plotted as the green dashed and blue solid curves, respectively. The anisotropy shifts the magnon-polaron peak positions, but does not substantially modify their amplitude. On these grounds, we proceed with computing other transport coefficients for the configuration $\mathbf{H} \perp \nabla T$ only.

Figure 6(a) shows the magnon spin conductivity $\sigma_{x x}$ as function of the magnetic field and temperature for different values of $\eta$. Two peaks (dips) appear at $H_{\perp}$ and $H_{\|}$for $\eta=100$ $(\eta=0.01)$ at 10 and $50 \mathrm{~K}$, while they disappear for $\eta=1$. At very low temperatures, $T=1 \mathrm{~K}$, the anomalies are not visible anymore. The dependence of the spin conductivity on the temperature, on the angle between the magnetic field and temperature gradient, and on the scattering potentials ratio $\eta$ is the same as reported for the spin Seebeck coefficient $\zeta_{x x}$.

In Fig. 6(b), we plot the dependence of the magnon heat conductivity $\kappa_{x x}^{(\mathrm{m})}$ on the magnetic field and on the temperature for different values of $\eta$. The only difference with respect to the coefficient $\zeta_{x x}$ is in the ratio between the amplitudes of the two anomalies at $T=10 \mathrm{~K}$, at which the magnon modes contributing to the low-field $\left(H_{\perp}\right)$ anomaly are thermally excited, in contrast to high field $\left(H_{\|}\right)$modes. In $\zeta_{x x}$, the anomaly at $H_{\perp}$ should therefore by better visible, as is indeed the case. The magnon heat conductivity from Eq. (30) contains an additional factor in the integrand which is proportional to the energy of the magnon-polaron modes. The latter compensates for the lower thermal occupation, which explains why the anomaly at $H_{\|}$is more pronounced in comparison with the spin Seebeck effect.

Perhaps surprisingly, the total heat conductivity $\kappa_{x x}$ in Fig. 7(a) displays only dips for $\eta \neq 1$ at the special fields 

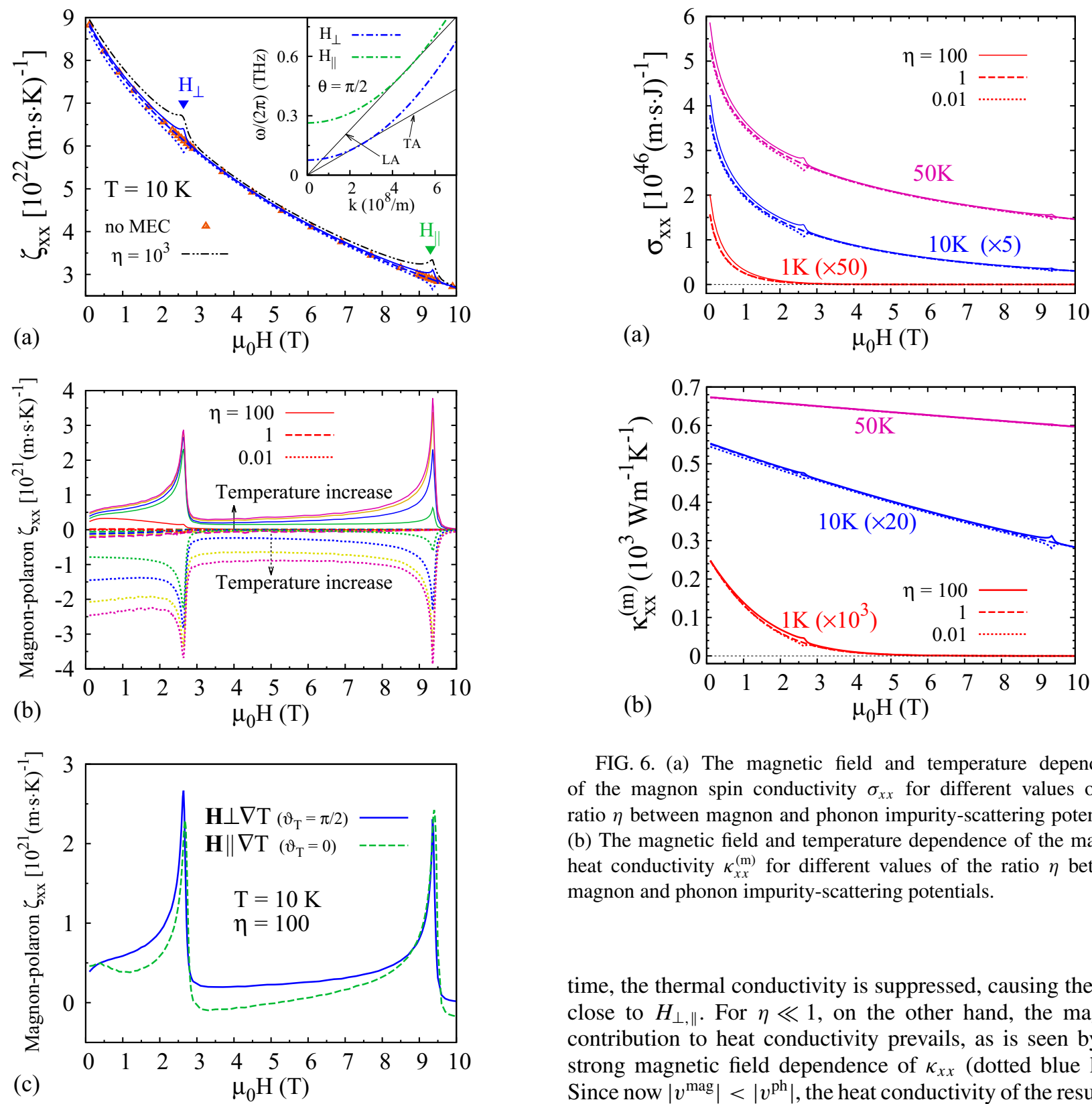

FIG. 5. (a) Spin Seebeck coefficient $\zeta_{x x}$ of bulk YIG as a function of magnetic field at $T=10 \mathrm{~K}$. The black dash-dotted, blue solid, blue dashed, blue dotted lines are computed for, respectively, $\eta=10^{3}, 100,1$, and 0.01 . The triangles are obtained for zero MEC. The inset shows the dispersions of uncoupled transverse (TA) and longitudinal (LA) acoustic phonons and the magnons shifted by $H_{\|}$ and $H_{\perp}$ magnetic fields. (b) The magnetic field and temperature dependence of the magnon-polaron contribution for different values of the ratio $\eta$ between magnon and phonon impurity-scattering potentials. (c) $\zeta_{x x}$ as function of magnetic field for $\mathbf{H} \perp \nabla T$ (blue solid line) and $\mathbf{H} \| \nabla T$ (green dashed line) at $T=10 \mathrm{~K}$ for $\eta=100$.

$H_{\perp, \|}$. This can be explained as follows. For $\eta \gg 1$, the phonon contribution to the heat conductivity is larger than the magnon contribution. Except at the critical fields $H_{\perp, \|}$, the magnetic field dependence of $\kappa_{x x}$ is therefore very weak (solid blue line). When phonons mix with magnons with a short scattering

FIG. 6. (a) The magnetic field and temperature dependence of the magnon spin conductivity $\sigma_{x x}$ for different values of the ratio $\eta$ between magnon and phonon impurity-scattering potentials. (b) The magnetic field and temperature dependence of the magnon heat conductivity $\kappa_{x x}^{(\mathrm{m})}$ for different values of the ratio $\eta$ between magnon and phonon impurity-scattering potentials.

time, the thermal conductivity is suppressed, causing the dips close to $H_{\perp, \|}$. For $\eta \ll 1$, on the other hand, the magnon contribution to heat conductivity prevails, as is seen by the strong magnetic field dependence of $\kappa_{x x}$ (dotted blue line). Since now $\left|v^{\mathrm{mag}}\right|<\left|v^{\mathrm{ph}}\right|$, the heat conductivity of the resulting magnon-polaron mode is lower than the purely magnonic one. Again dips appear close to the "touching" magnetic fields.

Experimentally, the magnon heat conductivity $\kappa_{x x}^{(\mathrm{m}, \exp )}$ at a given temperature was referred to the difference between finite-field value $\kappa_{x x}(H)$ and $\kappa_{x x}(\infty)$, i.e., $\kappa_{x x}^{(\mathrm{m}, \exp )}(H)=$ $\kappa_{x x}(H)-\kappa_{x x}(\infty)$ [24]. The latter, $\kappa_{x x}(\infty)$, corresponds to the saturation value of the heat conductivity at high-field limit, above which it becomes a constant function of the magnetic field, suggesting that the magnon contribution has been completely frozen out and only the phonon contribution remains. In general, $\kappa_{x x}^{(\mathrm{m})}$ and $\kappa_{x x}^{(\mathrm{m}, \exp )}$ differ in the presence of magnetoelasticity. The magnon heat conductivity $\kappa_{x x}^{(\mathrm{m}, \exp )}$ in Fig. 7(b), evaluated by subtracting the high-field limit for $T=10 \mathrm{~K}$, shows dips for both $\eta=0.01$ and 100 , in contrast to the magnon heat conductivity $\kappa_{x x}^{(\mathrm{m})}$ in Fig. 6(b) with peaks for $\eta=100$. The disagreement stems from $\kappa_{x x}(\infty)$, which is the (pure) phonon contribution to the heat conductivity at 
infinite magnetic fields, but is not the same as the phonon heat conductivity at ambient magnetic fields when the MEC is significant. In the latter case, the phonon heat conductivity itself depends on the magnetic field and displays anomalies at $H_{\perp, \|}$; hence $\kappa_{x x}^{(\mathrm{m}, \exp )} \neq \kappa_{x x}^{(\mathrm{m})}$.

Nonetheless, $\kappa_{x x}^{(\mathrm{m}, \exp )}$ can be useful since its fine structure contains information about the ratio between the magnonimpurity and phonon-impurity scattering potentials $\left|v^{\text {mag }}\right|$ and $\left|v^{\mathrm{ph}}\right|$. Also, $\kappa_{x x}(\infty)$ for $\eta=100$ is much larger than for $\eta=0.01$, and its value gives additional information about the relative acoustic and magnetic quality of the sample. For example, the results reported by Ref. [24] can be interpreted, within our theory, as suggesting a much higher acoustic than magnetic quality of the samples, i.e., $\eta \gg 1$. The authors, however, have not investigated the magnetic field dependence of the heat conductivity but rather the temperature dependence, which is beyond the scope of this work. It is worth to mention that already the work of Ref. [36] suggests that impurity scattering plays a key role in determining the magnetic field dependence of the heat conductivity.

The appearance of the anomalies can be understood analytically with few straightforward simplifications. Let us consider a one-dimensional system along $\hat{\mathbf{x}}$ and $\mathbf{H}=(0,0, H)$. According to Eq. (19) only the TA phonons couple to the magnons leading to the magnon-polaron dispersion

$$
\Omega_{1,2 k}=\frac{\omega_{k}+\omega_{1 k} \pm \sqrt{\left(\omega_{k}-\omega_{1 k}\right)^{2}+4 \tilde{\omega}_{k}^{2}}}{2},
$$

where $\tilde{\omega}_{k}=\left(S_{\perp} k\right)^{1 / 2}$ and $S_{\perp}=\left(n B_{\perp}\right)^{2}\left(\gamma \hbar^{2} / 4 M_{s} \bar{\rho} c_{\perp}\right)$. The magnon-polaron spin amplitudes $W_{1,2 k}$ are

$$
W_{1 k}=\frac{\omega_{k}-\omega_{1 k}+\sqrt{\left(\omega_{k}-\omega_{1 k}\right)^{2}+4 \tilde{\omega}_{k}^{2}}}{2 \sqrt{\left(\omega_{k}-\omega_{1 k}\right)^{2}+4 \tilde{\omega}_{k}^{2}}},
$$

and $W_{2 k}=1-W_{1 k}$. Disregarding the small dipolar interactions $\left(M_{s} \ll H_{\perp}\right)$ the uncoupled dispersions touch at $\mu_{0} H_{\perp}=$ $c_{\perp}^{2} / 4 D_{e x} \gamma$. We focus on the contribution of the $k_{\perp}$ mode (with $k_{\perp}=c_{\perp} / 2 D_{e x}$ ) to the transport coefficients (30) close to the touching field and expand in $\delta H=H-H_{\perp}$. As in Fig. 2(b), for $k=k_{\perp}$ and $\delta H \ll H_{\perp}$, the energies and group velocities of the upper and lower magnon-polarons are approximately the same, i.e., $\Omega_{1 k_{\perp}} \simeq \Omega_{2 k_{\perp}}$ and $\left.\partial_{k} \Omega_{1}\right|_{k=k_{\perp}} \simeq$ $\left.\partial_{k} \Omega_{2}\right|_{k=k_{\perp}}$. Equation (34) then reads

$$
W_{1 k_{\perp}}=\frac{1}{2}\left[1+\frac{\tilde{k} \delta H}{\sqrt{1+(\tilde{k} \delta H)^{2}}}\right],
$$

with $\tilde{k}=\mu_{0} \gamma /\left(4 S_{\perp} k_{\perp}\right)^{1 / 2}$. The scattering times (31) can be approximated as

$$
\tau_{1,2 k_{\perp}} \sim \frac{\left.\partial_{k} \Omega_{1,2 k}\right|_{k=k_{\perp}}}{\left|v_{\mathrm{ph}}\right|^{2}} \frac{1}{\left(1-W_{1,2 k_{\perp}}\right)+\eta W_{1,2 k_{\perp}}} .
$$

Hence

$$
\begin{aligned}
L_{x x}^{n m} \sim & \left.\frac{\beta}{L^{2}\left|v_{\mathrm{ph}}\right|^{2}}\left(\partial_{k} \Omega_{1 k}\right)^{3} \frac{e^{\beta \hbar \Omega_{1 k}}}{\left(e^{\beta \hbar \Omega_{1 k}}-1\right)^{2}}\left(\hbar \Omega_{1 k}\right)^{n}\right|_{\substack{k=k_{\perp}, H=H_{\perp}}} \\
& \times y_{m}(\delta H),
\end{aligned}
$$


FIG. 7. (a) The magnetic field dependence of the heat conductivity $\kappa_{x x}$ at $T=10 \mathrm{~K}$ for different scattering parameters $\eta$. (b) Magnetic field dependence of the heat conductivity difference $\kappa_{x x}(H)-\kappa_{x x}(\infty)$ simulating the experimental procedure [24] at $T=10 \mathrm{~K}$.

where

$$
y_{0}(\delta H)=\frac{4\left[1+(\tilde{k} \delta H)^{2}\right](1+\eta)}{1+\eta\left[2+4(\tilde{k} \delta H)^{2}+\eta\right]}
$$

and

$$
y_{1}(\delta H)=\frac{2\left[1+2(\tilde{k} \delta H)^{2}+\eta\right]}{1+\eta\left[2+4(\tilde{k} \delta H)^{2}+\eta\right]} .
$$

The indices $n$ and $m$ correspond to those in Eq. (30). Both $y_{0}(\delta H)$ and $y_{1}(\delta H)$ have a single extremum at $H=H_{\perp}$, i.e.,

$$
\begin{gathered}
\left.y_{0}^{\prime}(\delta H)\right|_{\delta H=0}=\left.y_{1}^{\prime}(\delta H)\right|_{\delta H=0}=0, \\
\left.y_{0}^{\prime \prime}(\delta H)\right|_{\delta H=0} \propto(1-\eta)^{2}, \\
\left.y_{1}^{\prime \prime}(\delta H)\right|_{\delta H=0} \propto(1-\eta) .
\end{gathered}
$$

Equations (38) and (39) prove that $y_{0}$ has a minimum at $H=H_{\perp}$ for $\eta \neq 1$, while for $\eta=1$ it is a constant. This explains our numerical results for the heat conductivity $\kappa_{x x}$, which is unstructured for $\eta=1$ and always display dips for both $\eta<1$ and $\eta>1$ [see Fig. 7(a)]. According to Eqs. (38) and (40) the function $y_{1}$ is also stationary at $H=H_{\perp}$, but it has a minimum only for $\eta<1$, while an inflection point for $\eta=1$, and a maximum otherwise. The resulting dependence on $\eta$ of Eq. (37) explains the spin Seebeck coefficient $\zeta_{x x}$, the 
spin conductivity $\sigma_{x x}$ and magnon heat conductivity $\kappa_{x x}^{(\mathrm{m})}$, in Figs. 4, 6(a), and 6(b), respectively. As we have discussed in detail in the reporting of the numerical results, the anomalies can be understood physically in terms of the scattering time of the magnon-polaron. This scattering time is the sum of magnonic and phononic scattering times, so, depending on the value of $\eta$, the spin transport is enhanced $(\eta>1)$ or suppressed $(\eta<1)$ close to the touching point.

\section{B. Spin diffusion length}

Integrating the spin-projection of Eq. (24) over momentum leads to the spin conservation equation:

$$
\dot{n}_{s}+\nabla \cdot j_{s}=-g_{\mu} \mu,
$$

where

$$
n_{s}=\int \frac{d^{3} \mathbf{k}}{(2 \pi)^{3}} \sum_{i} f_{i \mathbf{k}}(\mathbf{r}),
$$

is the total magnon density (in units of $\hbar$ ), and

$$
g_{\mu}=\beta \int \frac{d^{3} \mathbf{k}}{(2 \pi)^{3}} \sum_{i} W_{i \mathbf{k}} \frac{1}{\tau_{i \mathbf{k}}^{\mathrm{nc}}} \frac{e^{\beta \hbar \Omega_{i \mathbf{k}}}}{\left(e^{\beta \hbar \Omega_{i \mathbf{k}}}-1\right)^{2}}
$$

is the magnon relaxation rate, and we have introduced the relaxation time $\tau_{i \mathbf{k}}^{\mathrm{nc}}$. Elastic magnon-impurity scattering processes discussed in the previous sections do not contribute to $\tau_{i \mathbf{k}}^{\mathrm{nc}}$. However, we parametrize the spin not-conserving processes as

$$
\frac{1}{\tau_{i \mathbf{k}}^{\mathrm{nc}}}=2 \alpha \Omega_{\mathbf{k} i},
$$

in terms of the dimensionless Gilbert damping constant $\alpha$. In the nonequilibrium steady-state, Eq. (41) becomes

$$
\nabla^{2} \mu=\frac{1}{\lambda_{n}} \mu
$$

in terms of the magnon diffusion length $\lambda_{n} \equiv \sqrt{\sigma_{x x} / g_{\mu}}$ that is plotted in Fig. 8. At $10 \mathrm{~K}$ and $50 \mathrm{~K}$, the spin diffusion length decreases monotonously with the magnetic field for $\eta=1$, in agreement with observations at room temperature [37]. For $\eta=100(\eta=0.01)$ the spin diffusion length displays two peaks (dips) at the critical fields $H_{\perp}$ and $H_{\|}$, which become more pronounced when lowering the temperature. At $T=1 \mathrm{~K}$, only the peak (dip) at $H_{\perp}$ is visible for $\eta=100(\eta=0.01)$. For $\eta=1$, the spin diffusion length monotonically decreases with increasing magnetic field. The curve for $\eta=0.01$ behaves similarly except for the $\operatorname{dip}$ at $H=H_{\perp}$. On the other hand, for $\eta=100$, the spin diffusion length behaves very differently showing strong enhancement at both low and high magnetic fields.

This strong increase of the diffusion length (for constant Gilbert damping) happens when

$$
\frac{\sigma_{x x}\left(H_{1}, \eta\right)}{\sigma_{x x}\left(H_{2}, \eta\right)}>\frac{g_{\mu}\left(H_{1}\right)}{g_{\mu}\left(H_{2}\right)},
$$

where $H_{1,2}$ are two given values of the applied magnetic field, with $H_{1}>H_{2}$. To understand the dependence of the ratio $\sigma_{x x}\left(H_{1}, \eta\right) / \sigma_{x x}\left(H_{2}, \eta\right)$ on $\eta$ and on the temperature, we

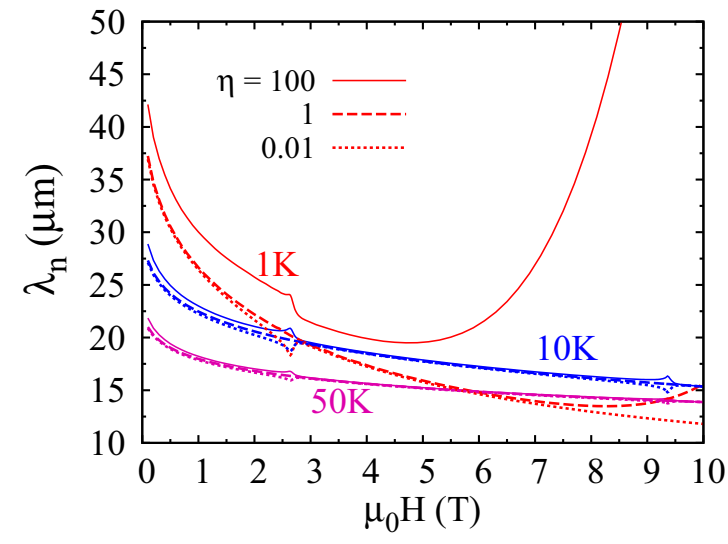

FIG. 8. Magnetic field and temperature dependence of the magnon diffusion length $\lambda_{n}$ for different values of the scattering parameter $\eta$.

recall that the main contribution to the magnon spin conductivity $\sigma_{x x}$ arises from magnonlike branches. At relatively high temperature, the magnonlike branches are sufficiently populated to overcome the phonon contribution to the magnon spin conductivity at all $\eta$. Indeed, Fig. 6(a) shows that, at relatively high temperatures, the ratio $\sigma_{x x}\left(H_{1}, \eta\right) / \sigma_{x x}\left(H_{2}, \eta\right)$ hardly depends on $\eta$. On the other hand, when the temperature decreases below the magnon energy, the contribution of the magnon-like branches are quickly frozen out by a magnetic field. The magnitude of $\eta$ then becomes very relevant. On the other hand, while the right-hand side of Eq. (46) depends on temperature, it is not affected by $\eta$. For $\eta<1$, the phonon mobility is smaller than the magnon one and hence the phonons are short circuited by the magnons. For $\eta>1$, the phonons prevail, leading to a higher ratio $\sigma_{x x}\left(H_{1}, \eta\right) / \sigma_{x x}\left(H_{2}, \eta\right)$ because the phonon dispersion is not affected by the magnetic field. When $\eta \gg 1$, the condition (46) is therefore satisfied. While in this regime the spin current is very small, it is perhaps an interesting limit for studying fluctuation and shot noise in the spin current [9].

\section{Comparison with experiments}

The spin Seebeck effect was measured in Pt|YIG|GGG structures in the longitudinal configuration, i.e., by applying a temperature difference normal to the interfaces ( $x$ direction) and subjecting the sample to a magnetic field $\mathbf{H} \| \hat{\mathbf{z}}$ [12]. The thermal bias induces a spin current into the Pt layer that by the inverse spin Hall effect (ISHE) leads to the detected transverse voltage $V$ over the contact, see Fig. 1. The bottom of the GGG substrate and the top of the Pt layer are in contact with heat reservoirs at temperature $T_{L}$ and $T_{H}$, respectively. Disregarding phonon (Kapitza) interface resistances, the phonon temperature gradient is $\nabla T=\left(T_{H}-T_{L}\right) / L$, with $L$ being the thickness of the stack, and average temperature $T=\left(T_{H}+T_{L}\right) / 2$. As discussed, we assume that the magnon and phonon temperatures are the same and disregard the interface mixing conductance. The measured voltage is then directly proportional to the bulk spin Seebeck coefficient.

In the experimental temperature range of $3.5-50 \mathrm{~K}$ the thermal magnon, $\Lambda_{\mathrm{mag}}$, and phonon, $\Lambda_{\mathrm{ph}, \mu}$, wavelengths are 
of the order of 1-10 $\mathrm{nm}$. Even if the magnon and phonon thermal mean free paths have been estimated to be of the order of $\sim 100 \mu \mathrm{m}$ at very low temperatures [24], here we assume that the transport in the YIG film of thickness $L \simeq 4 \mu \mathrm{m}$ can be treated semiclassically. Note that scattering at the interfaces can make the transport diffusive even when the formal conditions for diffusive transport are not satisfied. The bulk spin Seebeck coefficient is then well-described by Eq. (30) and proportional to the observed voltage $V$. These assumptions are encouraged by the good agreement for the observed and calculated peak structures at $H_{\perp}$ and $H_{\|}$with a single fitting parameter $\eta=100$ [12]. We may therefore conclude that the magnons are more strongly scattered than the phonons. This points towards a relatively high crystalline quality of the sample and towards the presence of magnetic impurities. Experimentally [38], it was indeed found that that the magnon and phonon scattering rate could be relatively tuned, which provides a possible route to experimentally investigate our predictions.

\section{CONCLUSION AND OUTLOOK}

We have established a framework which captures the effects of the magnetoelastic interaction on the transport properties of magnetic insulators. In particular, we show that the magnonphonon coupling gives rise to peaklike or diplike structures in the field dependence of the spin and heat transport coefficients, and of the spin diffusion length.

Our numerical evaluation reproduces the peaks in the observed low temperature longitudinal spin Seebeck voltages of YIG | Pt layers as a function of magnetic field. We quantitatively explain the temperature-dependent behavior of these anomalies in terms of hybrid magnon-phonon excitations ("magnon-polarons"). The peaks occur at magnetic fields and wave numbers at which the phonon dispersion curves are tangents to the magnon dispersion, i.e., when magnon and phonon energies as well as group velocities become the same. Under these conditions the effects of the magnetoelastic interaction are maximized. The computed angle dependence shows a robustness of the anomalies with respect to rotations of the magnetization relative to the temperature gradient. The agreement between the theory and the experimental results confirms that elastic magnon(phonon) impurity scattering is the main relaxation channel that limits the low temperature transport in YIG. Our theory contains one adjustable parameter that is fitted to the large set of experimental data, consistently finding a much better acoustic than magnetic quality of the samples. The spin Seebeck effect is therefore a unique analytical instrument not only of magnetic, but also mechanical material properties. The predicted effects of magnon-polaron effects on magnonic spin and heat conductivity call for further experimental confirmation.

We believe that the presented results open new avenues in spin caloritronics. We focused here on the low energy magnon dispersion of cubic YIG, which is well represented by the magnetostatic exchange waves of a homogeneous ferromagnet [21]. However, the theoretical framework can be easily extended to include anisotropies as well as ferrior antiferromagnetic order. The magnetoelastic coupling in YIG is relatively small and the conspicuous magnon-polaron effects can be destroyed easily. However, in materials with large magnon-phonon couplings these effects should survive in the presence of larger magnetization broadening as well as higher temperatures.

\section{ACKNOWLEDGMENTS}

This work was supported by the Stichting voor Fundamenteel Onderzoek der Materie (FOM), the European Research Council (ERC), the DFG Priority Programme 1538 "Spin-Caloric Transport", Grant-in-Aid for Scientific Research on Innovative Area "Nano Spin Conversion Science" (Grants No. JP26103005 and No. JP26103006), Grant-in-Aid for Scientific Research (A) (Grants No. JP25247056 and No. JP15H02012), and (S) (Grant No. JP25220910) from JSPS KAKENHI, Japan, PRESTO "Phase Interfaces for Highly Efficient Energy Utilization" and ERATO "Spin Quantum Rectification Project" from JST, Japan, NEC Corporation, and The Noguchi Institute. It is part of the D-ITP consortium, a program of the Netherlands Organization for Scientific Research (NWO) that is funded by the Dutch Ministry of Education, Culture and Science (OCW). T.K. is supported by JSPS through a research fellowship for young scientists (Grant No. 15J08026).
[1] E. Abrahams and C. Kittel, Phys. Rev. 88, 1200 (1952); Rev. Mod. Phys. 25, 233 (1953).

[2] C. Kittel, Phys. Rev. 110, 836 (1958).

[3] T. Kobayashi, R. C. Barker, J. L. Bleustein, and A. Yelon, Phys. Rev. B 7, 3273 (1973).

[4] M. I. Kaganov and V. M. Tsukernik, Sov. Phys. JETP 9, 151 (1959).

[5] G. E. W. Bauer, E. Saitoh, and B. J. van Wees, Nat. Mater. 11, 391 (2012).

[6] A. Rückriegel, P. Kopietz, D. A. Bozhko, A. A. Serga, and B. Hillebrands, Phys. Rev. B 89, 184413 (2014).

[7] M. Weiler, H. Huebl, F. S. Goerg, F. D. Czeschka, and R. Gross, and S. T. B. Goennenwein, Phys. Rev. Lett. 108, 176601 (2012).

[8] K. Uchida, H. Adachi, T. An, H. Nakayama, M. Toda, B. Hillebrands, S. Maekawa, and E. Saitoh, J. Appl. Phys. 111, 053903 (2012).
[9] A. Kamra, H. Keshtgar, P. Yan, and G. E. W. Bauer, Phys. Rev. B 91, 104409 (2015).

[10] N. Ogawa, W. Koshibae, A. J. Beekman, N. Nagaosa, M. Kubota, M. Kawasaki, and Y. Tokura, Proc. Natl. Acad. Sci. USA 112, 8977 (2015).

[11] K. Shen and G. E. W. Bauer, Phys. Rev. Lett. 115, 197201 (2015).

[12] T. Kikkawa, K. Shen, B. Flebus, R. A. Duine, K.-i. Uchida, Z. Qiu, G. E. W. Bauer, and E. Saitoh, Phys. Rev. Lett. 117, 207203 (2016).

[13] A. Akhiezer, V. Baryakhtar, and S. Peletminskii, Spin Waves (North Holland, Amsterdam, 1968).

[14] V. Cherepanov, I. Kolokolov, and V. L'vov, Phys. Rept. 229, 81 (1993).

[15] I. S. Tupitsyn, P. C. E. Stamp, and A. L. Burin, Phys. Rev. Lett. 100, 257202 (2008). 
[16] T. Holstein and H. Primakoff, Phys. Rev. 58, 1098 (1940).

[17] F. Keffer, Handbuch der Physik (Springer Verlag, Berlin, 1966), Vol. 18, p. 1.

[18] E. Schlömann, J. Appl. Phys. 31, 1647 (1960).

[19] E. M. Lifshitz and L. P. Pitaevshkii, Statistical Physics (Elsevier, Singapore, 2007).

[20] R. Damon and J. Eshbach, J. Phys. Chem. Solids 19, 308 (1961).

[21] J. Barker and G. E. W. Bauer, Phys. Rev. Lett. 117, 217201 (2016).

[22] C. Kittel, Quantum Theory of Solids (Willey, New York, 1963).

[23] J. Colpa, Physica A 93, 327 (1978).

[24] S. R. Boona and J. P. Heremans, Phys. Rev. B 90, 064421 (2014).

[25] M. A. Gilleo and S. Geller, Phys. Rev. 110, 73 (1958).

[26] A. B. Harris, Phys. Rev. 132, 2398 (1963).

[27] S. A. Manuilov, S. I. Khartsev, and A. M. Grishin, J. Appl. Phys. 106, 123917 (2009).

[28] C. M. Srivastava and R. Aiyar, J. Phys. C 20, 1119 (1987).
[29] W. Strauss, in Physical Acoustics, Principles and Methods, edited by W. Mandson (Academic Press, New York, 1967), Vol. 4, pt. B.

[30] A. G. Gurevich and G. A. Melkov, Magnetization Oscillations and Waves (CRC Press, Boca Raton, 1996).

[31] F. G. Eggers and W. Strauss, J. Appl. Phys. 34, 1180 (1963).

[32] P. Hansen, Phys. Rev. B 8, 246 (1973).

[33] L. J. Cornelissen, K. J. H. Peters, G. E. W. Bauer, R. A. Duine, and B. J. van Wees, Phys. Rev. B 94, 014412 (2016).

[34] T. Kikkawa, K.-i. Uchida, S. Daimon, Z. Qiu, Y. Shiomi, and E. Saitoh, Phys. Rev. B 92, 064413 (2015).

[35] H. Jin, S. R. Boona, Z. Yang, R. C. Myers, and J. P. Heremans, Phys. Rev. B 92, 054436 (2015).

[36] R. L. Douglass, Phys. Rev. 129, 1132 (1963).

[37] L. J. Cornelissen, and B. J. van Wees, Phys. Rev. B 93, 020403(R) (2016).

[38] S. J. Watzman, R. A. Duine, Y. Tserkovnyak, S. R. Boona, H. Jin, A. Prakash, Y. Zheng, and J. P. Heremans, Phys. Rev. B 94, 144407 (2016). 